\title{
Don't help them to bury the light. The interplay between intersystem crossing and hydrogen transfer in photoexcited curcumin revealed by state-hopping dynamics. ${ }^{\dagger}$
}

\author{
Raul Losantos, ${ }^{\mathrm{a}, \mathrm{b}}$ Andreea Pasc, ${ }^{\mathrm{b}}$ and Antonio Monaria,c,* \\ Curcumin is a natural compound extracted from turmeric (curcuma longa), which has shown remarkable antiinflammatory, \\ antibacterial, and possibly anticancert properties. The intense absorption in the visible domain, and the possibility of \\ intersystem crossing make curcumin attractive also for photodynamic therapy purposes. In the present contribution we \\ unravel, thanks to non-adiabatic state hopping dynamics, the competition between intersystem crossing and hydrogen \\ transfer within enol, the most stable tautomer of curcumin. Most notably, we show that while hydrogen-transfer is ultrafast \\ and happens in the sub-ps regime, intersystem crossing is still present, as shown by the non-negligible population of the \\ triplet state manifold after 2 ps. Hence, while the hydrogen transfer channel can act as an unfavorable deactivating channel, \\ curcumin, also in the red-shifted absorption enol form, can still be regarded as potentially favorable for photodynamic \\ therapy applications.
}

\section{Introduction}

Curcumin (CURC) is a natural compound, ${ }^{1}$ which is extracted from turmeric (curcuma longa) ${ }^{2}$ and is widely used as spice or food additive. The latter is also due to its intense orange colour, which makes curcumin particularly attractive for the food processing industry. More recently the interest in CURC also concerns its remarkable properties as antinflammatory and antibacterial drugs. ${ }^{1,3-7}$ Furthermore, it has been recently confirmed that curcumin exerts an important anticancer activity, ${ }^{8-10}$ which has further revived the pharmacological interest towards this natural compound. In addition, curcumin absorbing in the visible light, the possible exploitation of its excited state manifold for photodynamic (PDT) or light assisted chemo-therapy (LAC) have also been investigated.11,12 In particular, exploiting CURC, a natural occurring non-toxic spice, for PDT aimed at food processing and its antibacterial treatment is particularly attractive. Other analogue components of turmeric such as cyclocurcumin, have also been highlighted for their possible photophysical and photochemical properties. ${ }^{4,13-}$ 16

However, to ensure the efficiency of PDT, which is based on the activation of molecular oxygen to its reactive singlet state ${ }^{1} \mathrm{O}_{2}$, crucial photophysical conditions should be reunited. In particular, a high intersystem crossing (ISC), leading to a fast and efficient population of the photosensitizer triplet state manifold is required. Furthermore, the accessible triplet state should be energetic enough to allow sensitization of the molecular oxygen. Obviously, intense absorption in the visible, or even near infrared portion of the electromagnetic spectrum, together with a high bioavailability are also necessary to increase the global efficiency. Unfortunately, the last two properties are usually conflicting, since red-shifting the absorption spectrum usually requires the inclusion of large $\pi$ conjugated moieties, which in turn diminish solubility in aqueous media and biological conditions. As a matter of fact, the use of specific drug-delivering techniques for PDT agents, also based on their encapsulation in supramolecular aggregates or liposomes, has been proven successful to increase bioavailability and in vivo efficiency. ${ }^{17-20} \mathrm{~A}$ related advantage of tailored-drug delivery protocols relies on the possibility to functionalize the vector to enhance selectivity for cancer cell over healthy ones, hence adding up to the spatial selectivity brought by the use of light and hence minimize side-effects.

Moreover, to maximize the efficiency of PDT or LAC, and in general light-activated phenomena, the influence of nonradiative decay channels, such as thermal deactivation or photochemical isomerization, should be minimized to avoid quenching of the active excited state.

Hence, molecular modelling and simulation occupies a place of choice in the development of efficient PDT and LAC agents. Indeed, it allows to disentangle the complex interplay between opposite phenomena as well as the crucial influence of the inhomogeneous surrounding on the photophysical properties. ${ }^{17,21}$ In this sense molecular modelling and simulation are also invaluable to foster a more successful rational molecular design. 
Despite its non-negligible ${ }^{1} \mathrm{O}_{2}$ quantum yield, the complexity evoked is also clearly present for CURC. Indeed, natural CURC may exist in two tautomeric forms, namely keto and enol, which coexist in nature including upon administration into the human body. Recently, we have also shown that the inclusion in liposome or membranes, is susceptible to displace the equilibrium between the two forms, hence posing important burdens on the possibility of drug delivering strategies. ${ }^{22}$

A)

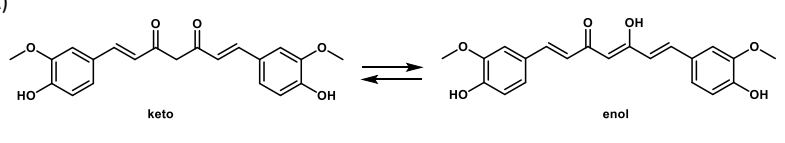

B)<smiles>COc1cc(/C=C/C=C2/C=C(/C=C/c3ccc(O)c(OC)c3)NO2)ccc1O</smiles>

Scheme 1. A) keto/enol equilibrium in CURC. B) $\mathrm{O}-\mathrm{H}$ distance defining the $\mathrm{H}$-transfer in enol CURC.

As shown in Scheme 1, the structural properties of keto and enol form are significantly different, which in turn leads to different optical and photophysical properties. ${ }^{22}$ Indeed, the enol tautomer is stabilized by an intermolecular hydrogen-bond ( $\mathrm{H}$-bond) between the $\mathrm{OH}$ and $\mathrm{C}=\mathrm{O}$ groups. Such interaction confers rigidity to the global structure, and more importantly leads to a highly planar scaffold, which favours delocalization and $\pi$-conjugation. As a consequence, enol CURC presents a considerable red-shifted absorption spectrum, which is highly beneficial for biological applications. Conversely, and also due to the $\mathrm{C}=\mathrm{O}$ lone pairs repulsion the keto tautomers shows a broken conjugation pattern and a twisted geometry, which correlates with a blue-shifted absorption. We have previously demonstrated, also through the proper exploration of the involved potential energy surfaces (PES), that both tautomers offer suitable, and energetically favourable, routes for ISC as well as possible fluorescence. ${ }^{22}$ However, the enol tautomer is usually regarded as unsuitable for PDT applications and ISC because of the opening of a relaxation pathways, based on the excited state transfer of the hydrogen involved in the intramolecular H-bond. ${ }^{23-25}$ Indeed, the lowest excited states of CURC having $\mathrm{n} \pi^{*}$ and $\pi \pi^{*}$ character they may lead to the weakening of the $\mathrm{OH}$ bond and hence favour the transfer.

In this contribution, going beyond our previous modelling, we resort to non-adiabatic molecular dynamics (NAMD) in the state-hopping ( $\mathrm{SH}$ ) formalism, ${ }^{26}$ to assess the competition between $\mathrm{H}$-transfer and ISC during the relaxation of the initially populated excited states. This is possible thanks to the inclusion of general coupling in the SH Hamiltonian, including spin orbit coupling (SOC) elements.

Our SH simulations clearly confirm the occurrence of the $\mathrm{H}$ transfer, already at a sub-ps timescale. However, we also show that ISC is not quenched and a significant, albeit non-dominant and non-ultrafast, population of the triplet state manifold is achieved.

\section{Results}

As previously stated, the equilibrium ground state of CURC is strongly dependent of its tautomer and indeed the enol form is highly rigid and planar. As shown in Figure $1 \mathrm{~A}$ an evident intramolecular $\mathrm{H}$-bond takes places stabilizing the planar arrangement of the chromophore. As a comparison the tilted structure of the keto tautomer is also reported in Figure 1B, even if in the following the latter will not be considered anymore.

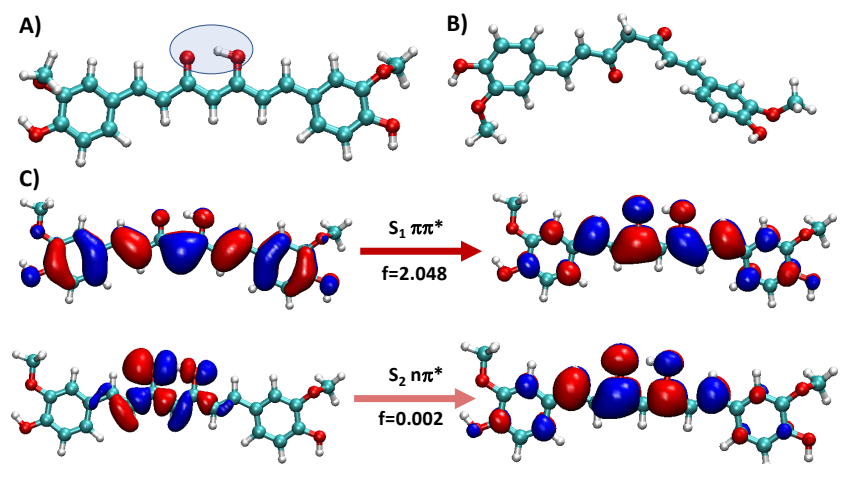

Figure 1. Optimized ground state structure for CURC in its enol (A) and keto (B) form NTOs for the two lowest excited states of enol tautomer of CURC showing the $\pi \pi^{*}$ (bright) and $n \pi^{*}$ (dark) nature of $\mathrm{S} 1$ and $\mathrm{S} 2$, respectively. The oscillator strength is also given for the two transitions and the intramolecular $\mathrm{H}$-bond is highlighted.

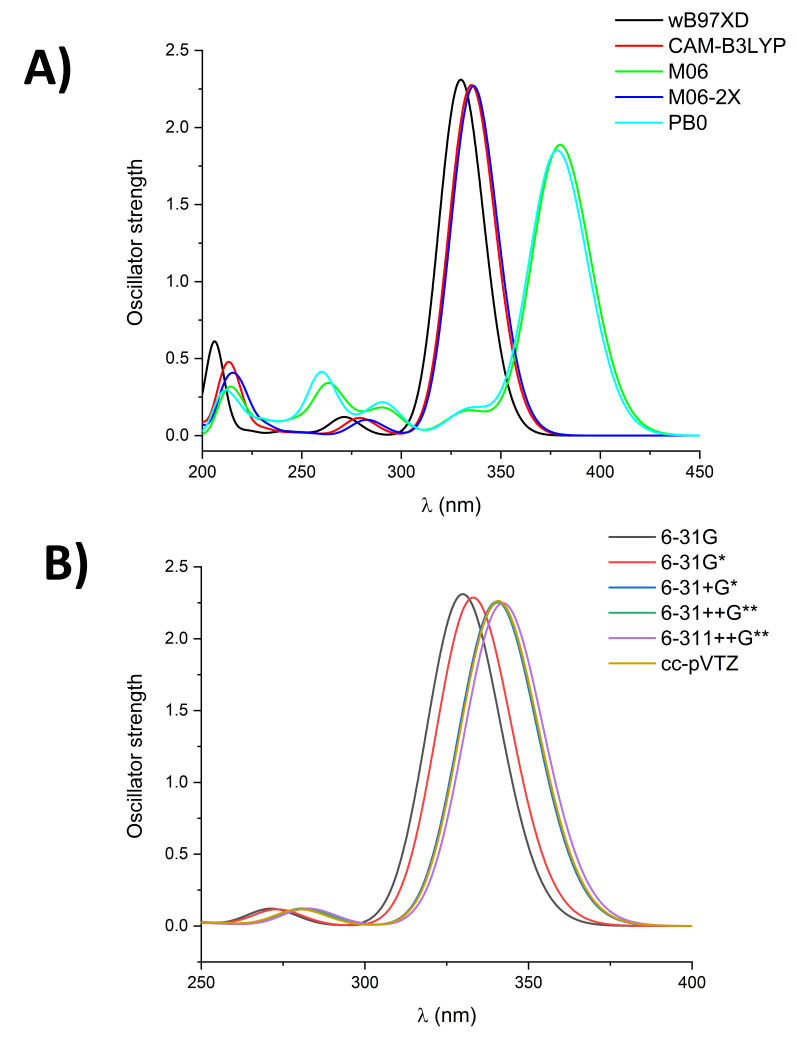

Figure 2. Absorption spectrum obtained as vertical transitions from the Franck Condon region using different functionals (A) and basis sets (B)

As far as the lowest lying excited states of enol CURC are considered and as shown in Figure $1 \mathrm{C}$ one can evidence the 
presence of a lower laying pp* state (S1), for which the symmetry allowed transition yields a high oscillator strength, and a darker $\mathrm{np}^{*}$ state (S2). Note that, coherently with what reported elsewhere the two states appears quasi-degenerate and have an excitation energy of about 3.7 and $3.9 \mathrm{eV}$, respectively. Note also that because of the high difference in the relative oscillator strength 2.048 vs 0.002 one may safely assume that the photophysics of enol CURC can be inferred considering the initial population of $\mathrm{S}_{1}$, only. An extended benchmark reporting the excitation energies calculated with different functionals and basis set can also be found in Electronic Supplementary Information (ESI). Note that, as shown in a previous contribution, the manifold of the singlet excited states for keto CURC will be more complicated due to the breaking of symmetry leading to an ensemble of mixed quasi-degenerate states having similar oscillator strength.

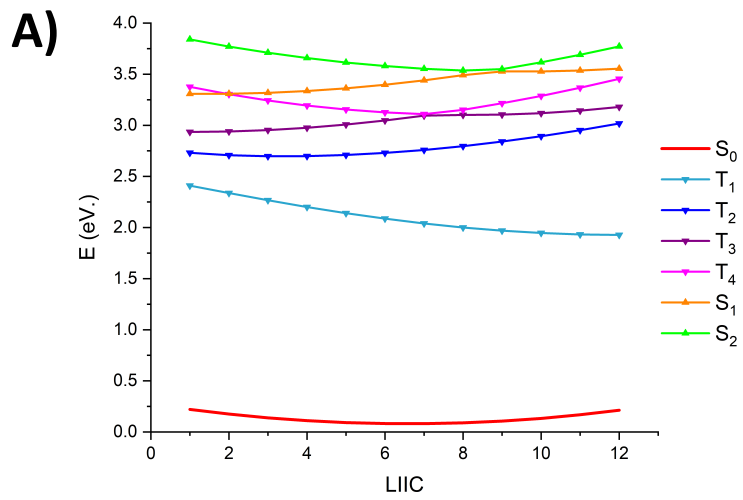

B)

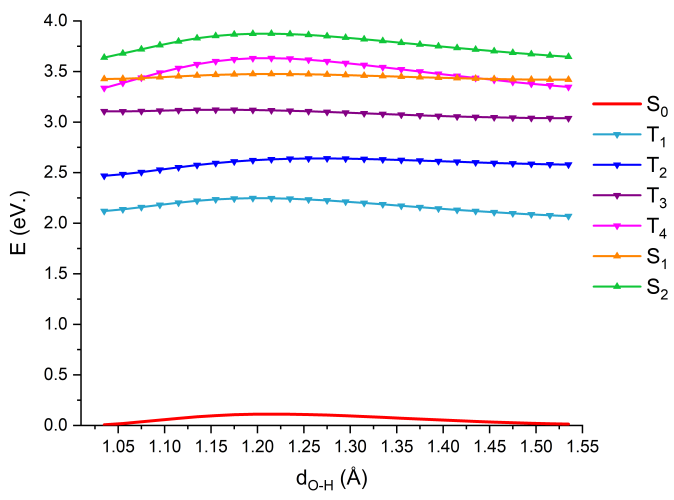

Figure 3. PES for the enol CURC along the LIIC connecting the equilibrium geometry of the first singlet and triplet (A). PES of the different excited state along the Hydrogen transfer coordinate (B).

As shown in Figure 2 the simulated absorption spectrum of enol CURC, calculated at TD-DFT level, is only moderately sensitive to the size and type of the basis set, hence justifying the use, especially for the subsequent SH dynamics the smaller 6-31G base. On the contrary, a more marked dependency on the functional nature and type can be observed with PBEO providing a highly red-shifted transition, while long-range corrected functionals behave coherently among them. In particular hybrid functional are also artificially lowering the energy of the $\mathrm{np}^{*}$ state, due to its partial charge-transfer character which can be appreciated from the low value of the $\phi_{\mathrm{s}}$ descriptor ( 0.3) indicating a small overlap between hole and electron densities. To further assess the effect of the functional and of the basis set on the general features of the PES we calculated the energies of triplet and singlet states at different critical point of the PES, namely Franck-Condon and $S_{1}$ and $T_{1}$ minima. The results are collected in ESI. Once again while the effect of the basis set appears negligible, the functional effects are more crucial. In any case the use of 6-31G basis in combination with the $\omega B 97 X D$ exchange-correlation functional provide a coherent description. In particular it evidences a manifold of low-lying states involving the first two excited singlet and the first four triplet states, which being close in energy can participate to the photophysical evolution upon excitation of the bright $\mathrm{S}_{1}$ state.

To further asses the coherence of the photophysical description with the reduced basis set we also report the analysis of the PES profile along the linear interpolation internal coordinate (LIIC) connecting the $\mathrm{S} 1$ and the $\mathrm{T} 1$ minima, i.e. along the collective variable driving ISC.

As shown in Figure $3 \mathrm{~A}$ we may indeed evidence that not only the $S_{1}$ state PES may cross one of the triplet $\left(T_{4}\right)$, but at its equilibrium region it also lays close in energy, and more importantly evolves in parallel, with the PES of T2 and T3. Such an arrangement, which is coherent with the one observed previously, may be indicative of a possible ISC, which however should not be ultrafast due to the necessity to overcome some barriers or to allow for the vibrational induced inversion between the energy of the quasi-degenerate singlet and triplet states. Note also that favourable ISC channel may exist also from S2, however due to the very low oscillator strength it is unlikely that they could play a major role in CURC photophysics. To complete the static analysis in Figure $3 \mathrm{~B}$ we also report the relaxed scan profile detailing the PES along the Hydrogen transfer coordinate. Interestingly, while most of the triplet states and $S_{2}$ presents a barrier, which can reach up to $0.2 / 0.3$ $\mathrm{eV}$, the process is barrierless on the $\mathrm{S}_{1}$ surface. Hence, the population of the $\mathrm{pp}^{*}$ excited state should certainly be connected with the weakening of the $\mathrm{OH}$ bond, and the strengthening of the complementary $\mathrm{H}$-bond, leading to the effective transfer.

If the H-transfer possibility is confirmed by the analysis of the $P E S$, it is also interesting to point out that the transfer itself does not seem to alter a favourable energetic order of states potentially leading to ISC crossing. Indeed, S1 PES is crossing twice a triplet state along the transfer coordinate and the general quasi-degeneracy with the triplet manifold is maintained.

Hence, while H-transfer should be considered as a clearly competitive process in the relaxation and de-excitation of enol CURC its final and global effect on the ISC rate and quantum yield appears less evident and necessitates a dynamic treatment to be properly taken into account.

The main results of the SH dynamic are summarized in Figure 4 and in ESI and show a rather complex behaviour confirming the interplay between ISC and $\mathrm{H}$-transfer. 


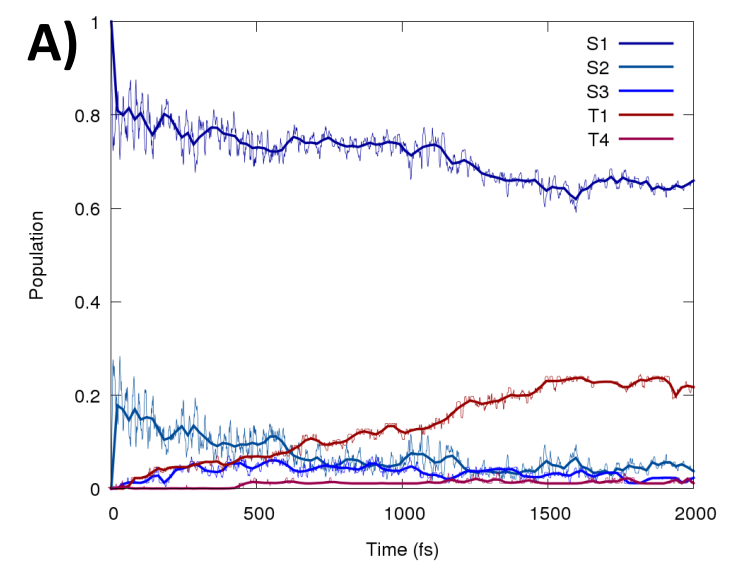

B)

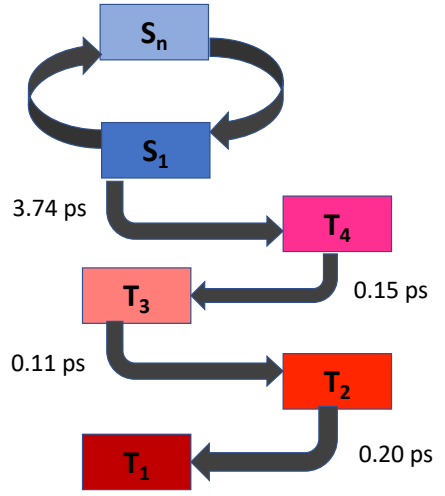

Figure 4. Evolution of the population of the states during the $\mathrm{SH}$ dynamic obtained from the multiconfiguration quantum amplitudes, triplet states are represented in red tones, convoluted for the ensemble of the trajectories. while singlets are in blue (A). Kinetic model for CURC ISC together with the fitted characteristic time $\tau$.

The evolution of the population of the different states (Figure $4 A)$ allows to underline some rather evident effects. Due to the difference in the oscillator strength we have considered that only the $S_{1}$ state will be populated after the initial excitation. However, after a few fs we may observe a redistribution of the population between $S_{1}$ and $S_{2}$ states, which is also due to the breaking of the symmetry and to a partial mixing of the $n \pi^{*}$ and $\pi \pi^{*}$ character, as also seen by the strong oscillation in the respective populations.

The transfer of population to the triplet state may be observed, at very modest rates, at around 50-100 fs. Interestingly, in this early stage the population seems to be driven mostly to the higher excited $\mathrm{T}_{4}$ state, which however are never showing a substantial population, also due to the very fast internal conversion (IC) to the lower triplet states. At larger time scales the population of $T_{1}$ becomes more important, especially after $500 / 600 \mathrm{fs}$. Interestingly the increase of the population of $T_{1}$ happens at similar time scales as the sharp decrease of the $S_{2}$ and a stabilization of $S_{1}$ population. As a matter of fact, at around $600 \mathrm{fs} T_{1}$ bypasses $S_{2}$ and at the end of the SH dynamics $(200 \mathrm{fs})$ it reaches a population of more than $20 \%$. Note that the other triplet states never achieve significant population and that a marginal participation of the higher singlet state $\mathrm{S}_{3}$ can also be observed.

This model is also confirmed by the analysis of the hops during the $\mathrm{SH}$ dynamics which points to a population flow from $\mathrm{S}_{1}$ to both $\mathrm{S}_{2}$ (as well as higher excited singlet states) and $\mathrm{T}_{4}$ (see ESI). Interestingly, while almost no transfer to the triplet population is observed from $\mathrm{S}_{2}$ a complex equilibrium involving singlet states up to $\mathrm{S}_{4}$ can be highlighted. On the other hand, after the initial population of $\mathrm{T}_{4}$ a very rapid interval conversion on the triplet manifold ensues leading to the sequential ultrafast population of $T_{3}, T_{2}$, and finally $T_{1}$. On these bases we may also sketch out a kinetic model (see Figure 4B) which yields out a rate-limiting $\mathrm{S}_{1} \rightarrow \mathrm{T}_{4}$ ISC characteristic time $\tau_{\text {ISC }}$ of $3.74 \mathrm{ps}$. On the contrary the cascade of IC on the triplet manifold takes place in the sub ps-range with characteristic time $\tau_{1 \mathrm{c}}$ comprised between 100 and $200 \mathrm{fs}$.
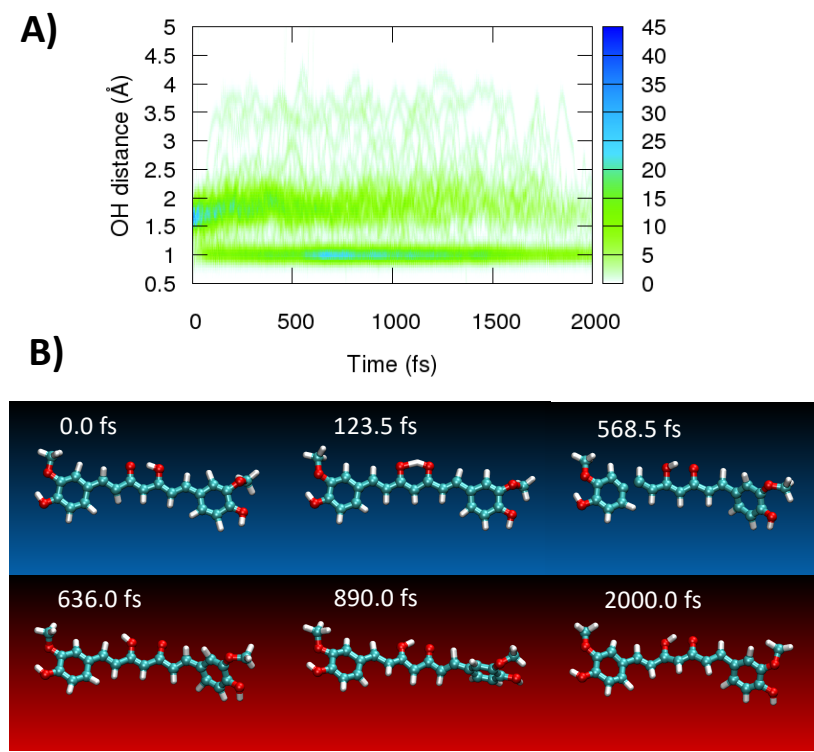

Figure 5. A) Evolution of the $\mathrm{OH}$ distance along the $\mathrm{SH}$ dynamic $\mathrm{B}$ ) representative snapshots of CURC for one trajectory leading to the ISC crossing at $569.0 \mathrm{fs}$ (note that the blue and red background indicates singlet or triplet state respectively).

As far as the $\mathrm{H}$-transfer is involved we analyse in Figure $5 \mathrm{~A}$ the distribution of the $\mathrm{O}-\mathrm{H}$ distance $\mathrm{d}$ highlighted in Scheme 1 on the ensemble of the $\mathrm{SH}$ trajectories and its time evolution. While for all the initial conditions d peaks at around $1.8 \AA$, which is coherent with a classical intramolecular $\mathrm{H}$-bond, even at the very first steps of the dynamic we may observe the branching of the distribution with the establishment of a cluster of trajectories presenting shorter distances of $0.9 \AA$, which are coherent with the formation of a novel covalent oxygen hydrogen bond. The flow of population between the two branches continues regularly along the $\mathrm{SH}$ population leading to an increase of the importance of the shorter distance structures which become dominant at around 600 ps. Interestingly, a continuous and bidirectional flux between the two populations can be observed all along the dynamic indicating the establishment of an equilibrium between the two tautomeric forms. Furthermore, in some cases we may observe a sudden 
increase of $d$ which may reach up to $4 \AA$ and show important oscillations. This fact can be associate to the activation of the out-of-plane rotation of the enol $\mathrm{OH}$ group. Finally, and as reported pictorially in Figure 5 we may observe $\mathrm{H}$-transfer both for trajectories leading to ISC and for trajectories remaining on the singlet state. Conversely, a significant breaking of the planarity can be observed for the $\mathrm{T}_{1}$ state as shown in Figure $5 \mathrm{~B}$. At the 2 ps limit, around $75 \%$ of the trajectories have transferred the involved hydrogen, and the phenomenon appeared for both trajectories leading to ISC or continuing exploring the singlet manifold.

\section{Materials and Methods}

The initial equilibrium geometries for the GS, as well as S1 and T1 states of enol CURC have been obtained at DFT level using the $\omega b 97 X^{27}$ functional together with the Pople double- $\zeta 6$ $31+G(d, p)$ basis set, coherently with what has been presented in Girardon et al. ${ }^{22}$ Water solvent has been implicitly considered using polarizable continuum method (PCM). ${ }^{28}$ In the case of the first singlet excited state TD-DFT has been used. All the calculations have been performed using the Gaussian09 suite of codes, harmonic vibrational frequencies have been obtained to assure the minimum nature of the stationary point. ${ }^{29}$

For benchmark purposes, the absorption spectrum has been calculated as vertical transitions from the GS using different functional namely, CAM-B3LYP,30 M06, M06-2X, ${ }^{31}$ PBE0,32 $\omega b 97 X D$, and different basis set of increasing size: 6-31G, 6$31 G(d), 6-31+G(d), 6-31++G(d, p), 6-311++G(d, p)$, and cc-pVTZ. The same levels of theory have also been used to assess the energetic order of the excited states, of both singlet and triplet multiplicity, at S1 and T1 geometries. The excited states have been calculated at TD-DFT level with the Tamm-Dancoff Approximation (TDA), ${ }^{33}$ which allows to avoid problems due ot triplet instability ${ }^{34}$ and hence provides a more balmanced description of the two multifold. As discussed in the Result section it appears that the combination of 6-31G and Wb97XD provides a reliable description of the PES, especially avoiding a computational burden, which will make render the subsequent SH dynamic sampling untreatable.

The nature of the excited states has been identified in terms of Natural Transition Orbitals (NTO) ${ }^{35}$ obtained via a postprocessing of the Gaussian output with the Nancy_Ex code. ${ }^{36}$ To better quantify the nature of the excited states we also calculated the topological fS index which provides the spatial overlap between hole and density matrices. ${ }^{36,37}$

To preliminary assess the possibility of $\mathrm{H}$-transfer and ISC crossing we also performed a relaxed scan at the 6$31 \mathrm{G} / \omega \mathrm{b} 97 \mathrm{XD}$ level along the $\mathrm{O}-\mathrm{H}$ distance $\mathrm{d}$ (see Scheme 1 and Figure 1) and we also explored the global degrees of freedom potentially leading to ISC by performing a linear interpolation of the internal coordinates to bridge $T_{1}$ and $S_{1}$ equilibrium geometries, using the same level of theory as for the relaxed scan. These calculations have been performed with Gaussian 09 code as well.

Finally, to assess the competition between the different relaxation routes and their timescales we performed in vacuo
SH dynamics using the Sharc code $26,38,39$ coupled with Orca. ${ }^{40}$ This strategy has already been used successfully by some of us on similar biological systems or photosensitizers. ${ }^{41-43}$ To this aim, 200 initial conditions around the Franck-Condon regions have been generated via a Wigner distribution, and the trajectories have been propagated at TD-DFT level, using the 6$31 \mathrm{G} / \omega \mathrm{b} 97 \mathrm{XD}$ level of theory, coherently with the previous benchmark. Due to their possible influence in the forthcoming dynamics we have calculated, in addition to the GS, 4 singlet excited states and 4 triplet states. SOC elements have been directly computed at each step of the dynamics with Orca, while the non-adiabatic coupling is approximated via the wavefunction overlap strategy. ${ }^{44}$ Considering the high difference in the oscillator strength, more than two order of magnitude, only the $\mathrm{S}_{1}$ state was initially populated. Each $\mathrm{SH}$ trajectory has been propagated with a time step of 0.5 fs to assure energy conservation for a total of $2 \mathrm{ps}$. The analysis of the population and distances have been performed on the ensemble of the trajectories, and the characteristic times have been obtained fitting the resulting evolution of the population to the established kinetic model.

The individual trajectories have been analysed and visualized also using the VMD code. ${ }^{45}$

\section{Conclusions}

CURC is a natural compound which can be promising for photosensitization purposes and more specifically for PDT and LAC applications. However, its complex, and environmental driven, conformational equilibrium may alter its native photophysical properties. Here we report a non-adiabatic molecular dynamic study of the behaviour of enol CURC after excitation to the bright $S_{1}$ state. After having properly benchmarked the chosen level of theory, achieving a good compromise between accuracy and the need of an extended sampling, we have shown that enol CURC may lead to ISC, albeit not in an ultrafast process and is characterized by a 3.4 ps rate determining step. ISC proceeds via the initial population of the high-laying $\mathrm{T}_{4}$ state and is followed by an ultrafast IC cascade in the triplet manifold. An equilibrium between $S_{1}$ and the higher excited states, especially $S_{2}$, and resulting in a population redistribution can also be evidenced.

In enol CURC ISC is also in competition between the excited state Hydrogen transfer that can happen via the intramolecular $\mathrm{H}$-bond relying the keto and enol group. This process is usually regarded as susceptible to quench ISC severely limiting its efficiency. Our SH dynamics have clearly shown the occurrence of the $\mathrm{H}$-transfer via a dynamic and ultrafast equilibrium. However, it has also been shown that triplet population is still possible, even in presence of this competitive mechanism. On the other hand, triplet population leads to a significant deformation of CURC skeleton, in particular breaking its planarity.

Our results help in rationalizing the complex photophysics of CURC and in particular the competition with other deactivating channels. As such it can help in the rational design of novel biomimetic sensitizers presenting enhanced ISC rates. If our 
results are unequivocal in pointing to the coexistence of $\mathrm{H}$ transfer and ISC, still are based on a rather minimal model, i.e. the photosensitizer in vacuo. The effect of the environment, either via electrostatic polarization or via the increased viscosity and mechanical resistance, can alter the profile of the involved PES and hence modify the final photophysical outcome. For this reason, in the following we plan to study the photophysical of CURC by hybrid quantum mechanics/molecular mechanic (QM/MM) SH dynamic explicitly including different environments such as water solution or lipid bilayers. ${ }^{46}$ On the same spirit, we also plan to consider the photophysics of the keto tautomer of CURC, in which the breaking of the $\pi$ conjugation leads to a manifold of bright singlet states which may be directly accessed upon excitation.

\section{Author Contributions}

A.M. conceived, planned and supervised the work and performed most of the SH dynamic. R.L. performed the benchmark calculations and the static exploration of the PES. A.P. participated to the planning and supervision of the work. A.M. prepared the first draft of the manuscript, which was corrected and approved by all the authors.

\section{Conflicts of interest}

There are no conflicts to declare.

\section{Acknowledgements}

Support from the Universite de Lorraine and CNRS is gratefully acknowledged. Calculations have been performed on the LPCT computing cluster and on the regional Explor computing center under the project "Dancing under the Light". Support from the European Union European Regional Development Funds (Programme opérationnel FEDER-FSE Lorraine et Massif des Vosges 2014-2020 "Fire Light" project: "Photo-bio-active molecules and nanoparticles") is also acknowledged.

\section{References}

S. Hewlings and D. Kalman, Curcumin: A Review of Its Effects on Human Health, Foods, 2017, 6, 92.

2 B. Sasikumar, in Handbook of Herbs and Spices: Second Edition, Elsevier Inc., 2012, vol. 1, pp. 526-546.

3 L. Wright, J. Frye, B. Gorti, B. Timmermann and J. Funk, Bioactivity of Turmeric-derived Curcuminoids and Related Metabolites in Breast Cancer, Curr. Pharm. Des., 2013, 19, 6218-6225.

4 Y. Li, M. Toscano, G. Mazzone and N. Russo, Antioxidant properties and free radical scavenging mechanisms of cyclocurcumin, New J. Chem., 2018, 42, 12698-12705.
V. Kuptniratsaikul, P. Dajpratham, W. Taechaarpornkul, M. Buntragulpoontawee, P. Lukkanapichonchut, C. Chootip, J. Saengsuwan, K. Tantayakom and S. Laongpech, Efficacy and safety of Curcuma domestica extracts compared with ibuprofen in patients with knee osteoarthritis: A multicenter study, Clin. Interv. Aging, 2014, 9, 451-458.

6 A. Altunbas, S. J. Lee, S. A. Rajasekaran, J. P. Schneider and D. J. Pochan, Encapsulation of curcumin in self-assembling peptide hydrogels as injectable drug delivery vehicles, Biomaterials, 2011, 32, 5906-5914.

M. D. Cas and R. Ghidoni, Dietary curcumin: Correlation between bioavailability and health potential, Nutrients, 2019, 11, 2147.

S. Hewlings and D. Kalman, Curcumin: A Review of Its' Effects on Human Health, Foods, 2017, 6, 92.

M. A. Tomeh, R. Hadianamrei and X. Zhao, A review of curcumin and its derivatives as anticancer agents, Int. J. Mol. Sci., 2019, 20, 1033.

J. Ravindran, S. Prasad and B. B. Aggarwal, Curcumin and cancer cells: How many ways can curry kill tumor cells selectively?, AAPS J., 2009, 11, 495-510.

A. Azizi, P. Shohrati, M. Goudarzi, S. Lawaf and A. Rahimi, Comparison of the effect of photodynamic therapy with curcumin and methylene Blue on streptococcus mutans bacterial colonies, Photodiagnosis Photodyn. Ther., 2019, 27, 203-209.

A. B. Ormond and H. S. Freeman, Dye sensitizers for photodynamic therapy, Materials (Basel)., 2013, 6, 817840.

M. Marazzi, A. Francés-Monerris, M. Mourer, A. Pasc and A. Monari, Trans -to- cis photoisomerization of cyclocurcumin in different environments rationalized by computational photochemistry, Phys. Chem. Chem. Phys., 2020, 22, 4749-4757.

T. Ngo, K. Kim, Y. Bian, G. J. An, O. N. Bae, K. M. Lim and J. $\mathrm{H}$. Chung, Cyclocurcumin from Curcuma longa selectively inhibits shear stress-induced platelet aggregation, J. Funct. Foods, 2019, 61, 103462.

R. Losantos, J. Pecourneau, M. Mourer, S. Parant, A. Pasc and $A$. Monari, trans-cisPhotoisomerization of a biomimetic cyclocurcumin analogue rationalized by molecular modelling, Phys. Chem. Chem. Phys., 2021, 23, 12842-12849.

J. Pecourneau, R. Losantos, A. Monari, S. Parant, A. Pasc and M. Mourer, Synthesis and Photoswitching Properties of Bioinspired Dissymmetric $\gamma$-Pyrone, an Analogue of Cyclocurcumin, J. Org. Chem., 2021, 86, 8112-8126. B. Aslanoglu, I. Yakavets, V. Zorin, H. P. Lassalle, F. Ingrosso, A. Monari and S. Catak, Optical properties of photodynamic therapy drugs in different environments: The paradigmatic case of temoporfin, Phys. Chem. Chem. Phys., 2020, 22, 16956-16964.

I. Yakavets, H. P. Lassalle, I. Yankovsky, F. Ingrosso, A. Monari, L. Bezdetnaya and V. Zorin, Evaluation of temoporfin affinity to $\beta$-cyclodextrins assuming selfaggregation, J. Photochem. Photobiol. A Chem., 2018, 367, 13-21.

J. Wang, M. Zheng and Z. Xie, Carrier-free core-shell nanodrugs for synergistic two-photon photodynamic therapy of cervical cancer, J. Colloid Interface Sci., 2019, 535, 84-91. 
C. S. Mangolim, C. Moriwaki, A. C. Nogueira, F. Sato, M. L. Baesso, A. M. Neto and G. Matioli, Curcumin- $\beta$-cyclodextrin inclusion complex: Stability, solubility, characterisation by FT-IR, FT-Raman, X-ray diffraction and photoacoustic spectroscopy, and food application, Food Chem., 2014, 153, 361-370.

M. Navarrete-Miguel, J. Segarra-Martí, A. FrancésMonerris, A. Giussani, P. Farahani, B.-W. Ding, A. Monari, Y.-J. Liu and D. Roca-Sanjuán, in Photochemistry: 46, 2018, pp. 28-77.

M. Girardon, S. Parant, A. Monari, F. Dehez, C. Chipot, E. Rogalska, N. Canilho and A. Pasc, Triggering Tautomerization of Curcumin by Confinement into Liposomes, ChemPhotoChem, 2019, 3, 1034-1041. T. Harada, S. F. Lincoln and T. W. Kee, Excited-state dynamics of the medicinal pigment curcumin in a hydrogel, Phys. Chem. Chem. Phys., 2016, 18, 28125-28133.

R. Ghosh, J. A. Mondal and D. K. Palit, Ultrafast dynamics of the excited states of curcumin in solution, J. Phys. Chem. B, 2010, 114, 12129-12143.

K. I. Priyadarsini, J. Photochem. Photobiol. C Photochem. Rev., 2009, 10, 81-95.

S. Mai, P. Marquetand and L. González, Nonadiabatic dynamics: The SHARC approach, Wiley Interdiscip. Rev. Comput. Mol. Sci., 2018, 8, e1370.

J.-D. Chai and M. Head-Gordon, Long-range corrected hybrid density functionals with damped atom-atom dispersion corrections, Phys. Chem. Chem. Phys., 2008, 10, 6615.

J. Tomasi, B. Mennucci and R. Cammi, Quantum mechanical continuum solvation models, Chem. Rev., 2005, 105, 2999-3093.

M. J. Frisch, G. W. Trucks, H. B. Schlegel, G. E. Scuseria, M. A. Robb, J. R. Cheeseman, G. Scalmani, V. Barone, G. A. Petersson, H. Nakatsuji, X. Li, M. Caricato, A. Marenich, J. Bloino, R. Janesko, R. Gomperts, B. Menucci, H. P. Hratchian, J. V. Ortiz, A. F. Izmaylov, D. Sonnenberg, F. Williams-Young, F. Ding, F. Lipparini, F. Edigi, J. Goings, B. Peng, A. Petrone, T. Henderson, D. Ranasinghe, V. G. Zakrzewski, J. Gao, N. Rega, W. Zheng, W. Liang, M. Hada, M. Ehara, K. Toyota, R. Fukuda, M. Hasegawa, T. Ishida, T. Nakajima, Y. Honda, O. Kitao, H. Nakai, T. Vreven, K. Throssell, M. J. J. A., J. E. Peralta, F. Ogliaro, M. Bearpark, J. J. Heyd, E. Brothers, K. N. Kudin, V. N. Staroverov, T. Keith, R. Kobayashi, J. Normand, K. Raghavachari, A. Rendell, J. C. Burant, S. S. Iyengar, J. Tomasi, M. Cossi, J. M. Millam, M. Klene, C. Adamo, R. Cammi, J. W. Ochterski, R. L. Martin, K. Morokuma, O. Farkas, J. B. Foresman, D. J. Fox, G. E. S. M. J. Frisch, G. W. Trucks, H. B. Schlegel, B. M. M. A. Robb, J. R. Cheeseman, G. Scalmani, V. Barone, H. P. H. G. A. Petersson, H. Nakatsuji, M. Caricato, X. Li, M. H. A. F. Izmaylov, J. Bloino, G. Zheng, J. L. Sonnenberg, T. N. M. Ehara, K. Toyota, R. Fukuda, J. Hasegawa, M. Ishida, J. Y. Honda, O. Kitao, H. Nakai, T. Vreven, J. A. Montgomery, E. B. J. E. Peralta, F. Ogliaro, M. Bearpark, J. J. Heyd, J. N. K. N. Kudin, V. N. Staroverov, T. Keith, R. Kobayashi, J. T. K. Raghavachari, A. Rendell, J. C. Burant, S. S. Iyengar, J. B. C.
M. Cossi, N. Rega, J. M. Millam, M. Klene, J. E. Knox, R. E. S. V. Bakken, C. Adamo, J. Jaramillo, R. Gomperts, J. W. O. O. Yazyev, A. J. Austin, R. Cammi, C. Pomelli, G. A. V. R. L. Martin, K. Morokuma, V. G. Zakrzewski, A. D. D. P. Salvador, J. J. Dannenberg, S. Dapprich, and D. J. F. O. Farkas, J. B. Foresman, J. V. Ortiz, J. Cioslowski, G. M. J. Frisch, W. Trucks, H. B. Schlegel, G. E. Scuseria, M. A. Robb, J. R. Cheeseman, G. Scalmani, V. Barone, B. Mennucci, G. A. Petersson, H. Nakatsuji, M. Caricato, X. Li, H. P. Hratchian, A. F. Izmaylov, J. Bloino, G. Zheng, J. L. Sonnenberg, M. J. Frisch, G. W. Trucks, H. B. Schlegel, G. E. Scuseria, M. A. Robb, J. R. Cheeseman, G. Scalmani, V. Barone, B.

Mennucci, G. A. Petersson, H. Nakatsuji, M. Caricato, X. Li, H. P. Hratchian, A. F. Izmaylov, J. Bloino, G. Zheng, J. L. Sonnenberg, M. Hada, M. Ehara, K. Toyota, R. Fukuda, J. Hasegawa, M. Ishida, T. Nakajima, Y. Honda, O. Kitao, H. Nakai, T. Vreven, J. A. Montgomery Jr., J. E. Peralta, F. Ogliaro, M. Bearpark, J. J. Heyd, E. Brothers, K. N. Kudin, V. N. Staroverov, R. Kobayashi, J. Normand, K. Raghavachari, A. Rendell, J. C. Burant, S. S. Iyengar, J. Tomasi, M. Cossi, N. Rega, J. M. Millam, M. Klene, J. E. Knox, J. B. Cross, V. Bakken, C. Adamo, J. Jaramillo, R. Gomperts, R. E. Stratmann, O. Yazyev, A. J. Austin, R. Cammi, C. Pomelli, J. W. Ochterski, R. L. Martin, K. Morokuma, V. G. Zakrzewski, G. A. Voth, P. Salvador, J. J. Dannenberg, S. Dapprich, A. D. Daniels, O. Farkas, J. B. Foresman, J. V. Ortiz, J. Cioslowski, D. J. Fox, W. C. Frisch, M. J.; Trucks, G. W.; Schlegel, H. B.; Scuseria, G. E.; Robb, M. A.; Cheeseman, J. R.; Scalmani, G.; Barone, V.; Mennucci, B.; Petersson, G. A.; Nakatsuji, H.; Caricato, M.; Li, X.; Hratchian, H. P.; Izmaylov, A. F.; Bloino, J.; Zheng, G.; Sonnenb, H. B. S. G. E. S. M. J. Frisch G. W. Trucks, G. S. V. B. B. M. M. A. Robb J. R. Cheeseman, M. C. X. L. H. P. H. G. A. Petersson H. Nakatsuji, G. Z. J. L. S. M. H. A. F. Izmaylov J. Bloino, R. F. J. H. M. I. T. N. M. Ehara K. Toyota, H. N. T. V. J. A. M. J. Y. Honda O. Kitao, M. B. J. J. H. E. B. J. E. Peralta F. Ogliaro, T. K. R. K. J. N. K. N. Kudin V. N. Staroverov, J. C. B. S. S. I. J. T. K. Raghavachari A. Rendell, J. M. M. M. K. J. E. K. J. B. C. M. Cossi N. Rega, J. J. R. G. R. E. S. V. Bakken C. Adamo, R. C. C. P. J. W. O. O. Yazyev A. J. Austin, V. G. Z. G. A. V. R. L. Martin K. Morokuma, S. D. A. D. D. P. Salvador J. J. Dannenberg, J. V. O. J. C. D. J. F. O. Farkas J. B. Foresman and D. J. Frisch, M. J.; Trucks, G.W.; Schlegel, H. B.; Scuseria, G. E.; Robb, M. A.; Cheeseman, J. R.; Scalmani, G.; Barone, V.;Mennucci, B.; Petersson, G. A.; Nakatsuji, H.; Caricato, M.; Li, X.; Hratchian, H. P.; Izmaylov, A. F.; Bloino, J.; Zheng, G.; Sonnenber, Gaussian 09, Gaussian 09 Revis. D.01, 2009, 2-3.

T. Yanai, D. P. Tew and N. C. Handy, A new hybrid exchange-correlation functional using the Coulombattenuating method (CAM-B3LYP), Chem. Phys. Lett., 2004, 393, 51-57.

31 Y. Zhao and D. G. Truhlar, The M06 suite of density functionals for main group thermochemistry, thermochemical kinetics, noncovalent interactions, excited states, and transition elements: Two new functionals and systematic testing of four M06-class functionals and 12 other function, Theor. Chem. Acc., 2008, 120, 215-241. 

functional methods without adjustable parameters: The PBE0 model, J. Chem. Phys., 1999, 110, 6158-6169.

S. Hirata and M. Head-Gordon, Time-dependent density functional theory within the Tamm-Dancoff approximation, Chem. Phys. Lett., 1999, 314, 291-299. M. J. G. Peach, M. J. Williamson and D. J. Tozer, Influence of triplet instabilities in TDDFT, J. Chem. Theory Comput., 2011, 7, 3578-3585.

R. L. Martin, Natural transition orbitals, J. Chem. Phys., 2003, 118, 4775-4777.

T. Etienne, X. Assfeld and A. Monari, Toward a quantitative assessment of electronic transitions" charge-transfer character, J. Chem. Theory Comput., 2014, 10, 3896-3905. T. Etienne, X. Assfeld and A. Monari, New Insight into the Topology of Excited States through Detachment/Attachment Density Matrices-Based Centroids of Charge, J. Chem. Theory Comput., 2014, 10, 3906-3914.

S. Mai, P. Marquetand and L. González, A general method to describe intersystem crossing dynamics in trajectory surface hopping, Int. J. Quantum Chem., 2015, 115, 12151231.

39 M. Richter, P. Marquetand, J. González-Vázquez, I. Sola and L. González, SHARC: Ab initio molecular dynamics with surface hopping in the adiabatic representation including arbitrary couplings, J. Chem. Theory Comput., 2011, 7, 1253-1258.

40 F. Neese, Software update: the ORCA program system, version 4.0, Wiley Interdiscip. Rev. Comput. Mol. Sci., 2018, 8, e1327.

M. Marazzi, S. Mai, D. Roca-Sanjuán, M. G. Delcey, R. Lindh, L. González and A. Monari, Benzophenone Ultrafast Triplet Population: Revisiting the Kinetic Model by SurfaceHopping Dynamics, J. Phys. Chem. Lett., 2016, 7, 622-626. A. Francés-Monerris, M. Lineros-Rosa, M. A. Miranda, V. Lhiaubet-Vallet and A. Monari, Photoinduced intersystem crossing in DNA oxidative lesions and epigenetic intermediates, Chem. Commun., 2020, 56, 4404-4407. M. Lineros-Rosa, A. Francés-Monerris, A. Monari, M. A. Miranda and V. Lhiaubet-Vallet, Experimental and theoretical studies on thymine photodimerization mediated by oxidatively generated DNA lesions and epigenetic intermediates, Phys. Chem. Chem. Phys., 2020, 22, 25661-25668.

F. Plasser, M. Ruckenbauer, S. Mai, M. Oppel, P. Marquetand and L. González, Efficient and Flexible Computation of Many-Electron Wave Function Overlaps, J. Chem. Theory Comput., 2016, 12, 1207-1219.

W. Humphrey, A. Dalke and K. Schulten, VMD: Visual molecular dynamics, J. Mol. Graph., 1996, 14, 33-38.

S. Mai, H. Gattuso, M. Fumanal, A. Muñoz-Losa, A. Monari, C. Daniel and L. González, Excited-states of a rhenium carbonyl diimine complex: Solvation models, spin-orbit coupling, and vibrational sampling effects, Phys. Chem. Chem. Phys., 2017, 19, 27240-27250. 


\section{Electronic Supplementary Information for:}

\section{Don't help them to bury the light. The interplay between intersystem crossing and hydrogen transfer in photoexcited curcumin revealed by state-hopping dynamics}

Raul Losantos, ${ }^{\mathrm{a}, \mathrm{b}}$ Andreea Pasc, ${ }^{\mathrm{b}}$ and Antonio Monari ${ }^{\mathrm{a}, \mathrm{c}, *}$

aUniversité de Lorraine and CNRS, UMR 7019 LPCT, F-54000 Nancy, France.

bUniversité de Lorraine and CNRS, UMR 7053 L2CM, F-54000 Nancy, France.

'Université de Paris and CNRS, Itodys, F-75006 Paris, France.

*Antonio.monari@univ-lorraine.fr
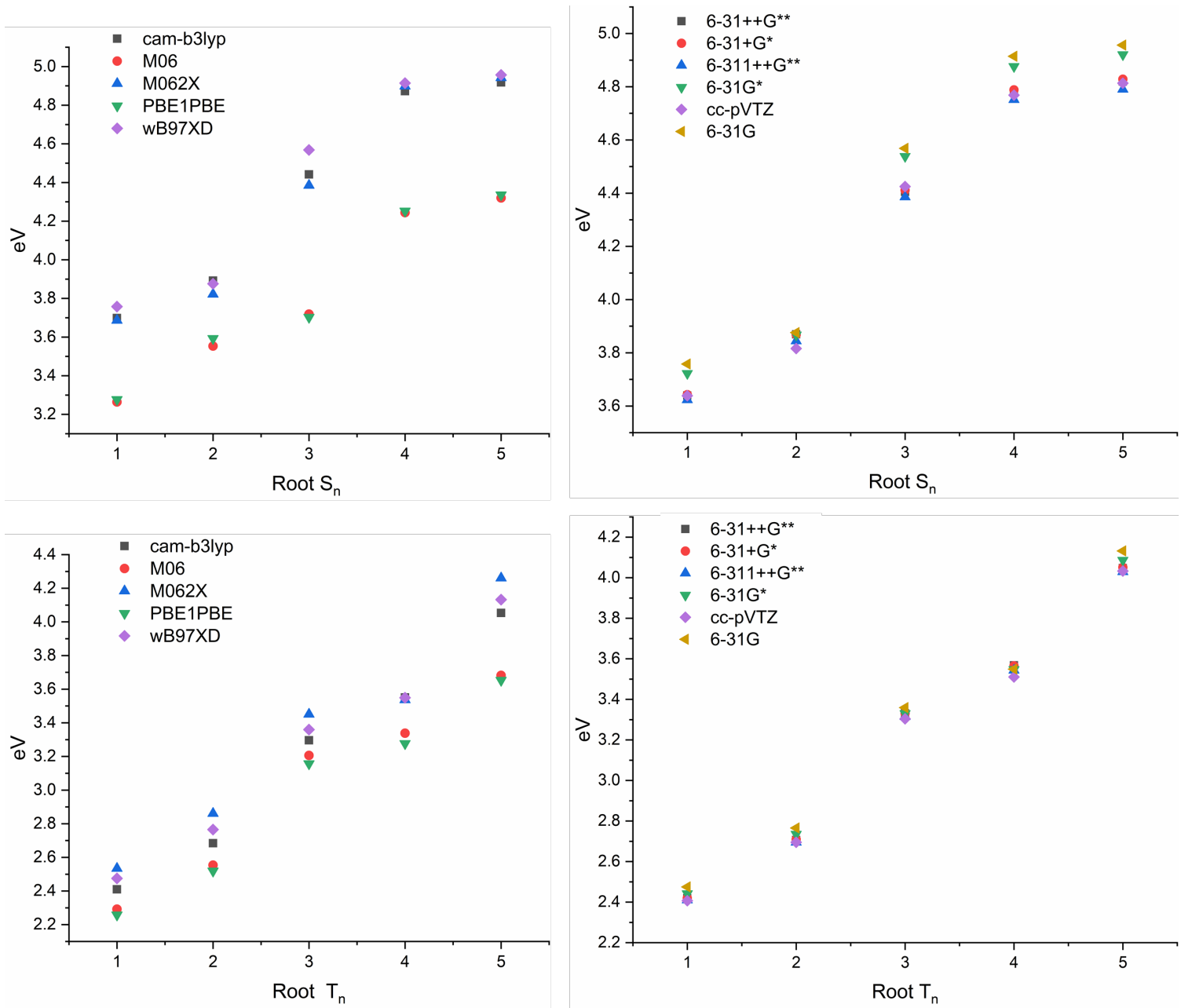

Figure S1. Energy levels of the excited singlet (top row) and triplet (bottom row) calculated with different functionals and basis sets at the Franck-Condon geometry. 

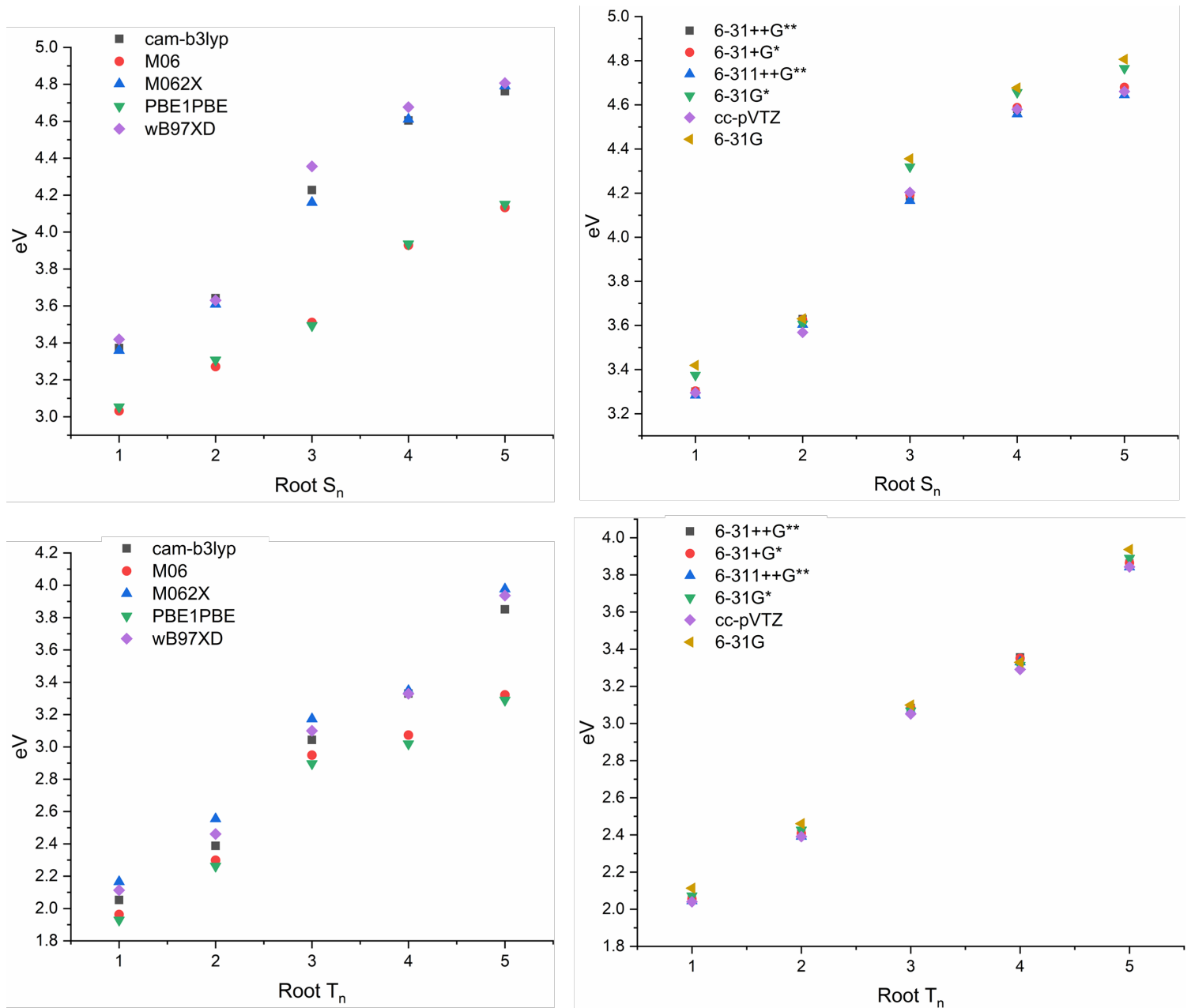

Figure S2. Energy levels of the excited singlet (top row) and triplet (bottom row) calculated with different functionals and basis sets at the $\mathrm{S}_{1}$ equilibrium geometry. 

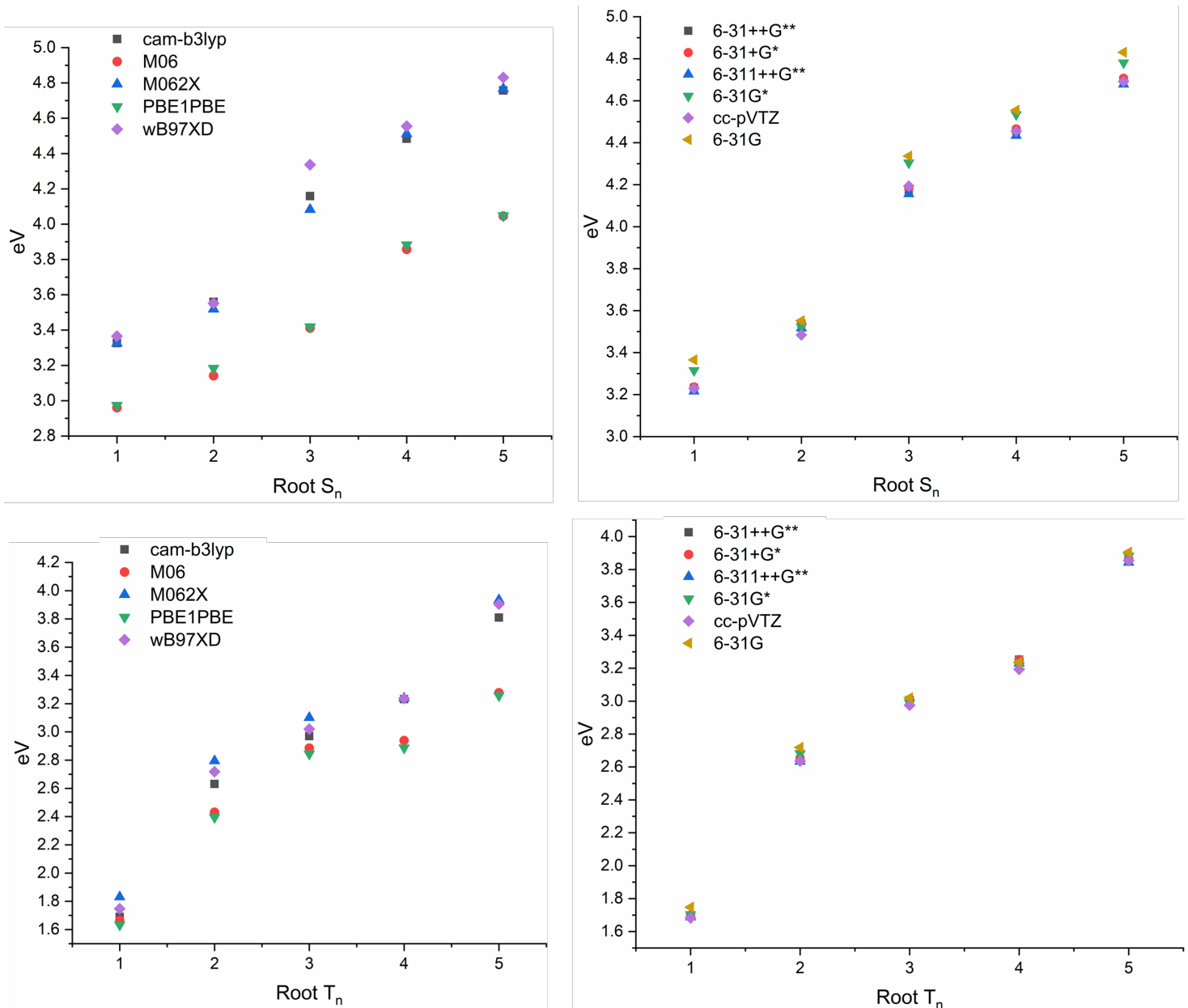

Figure S3. Energy levels of the excited singlet (top row) and triplet (bottom row) calculated with different functionals and basis sets at the $\mathrm{T}_{1}$ equilibrium geometry. 


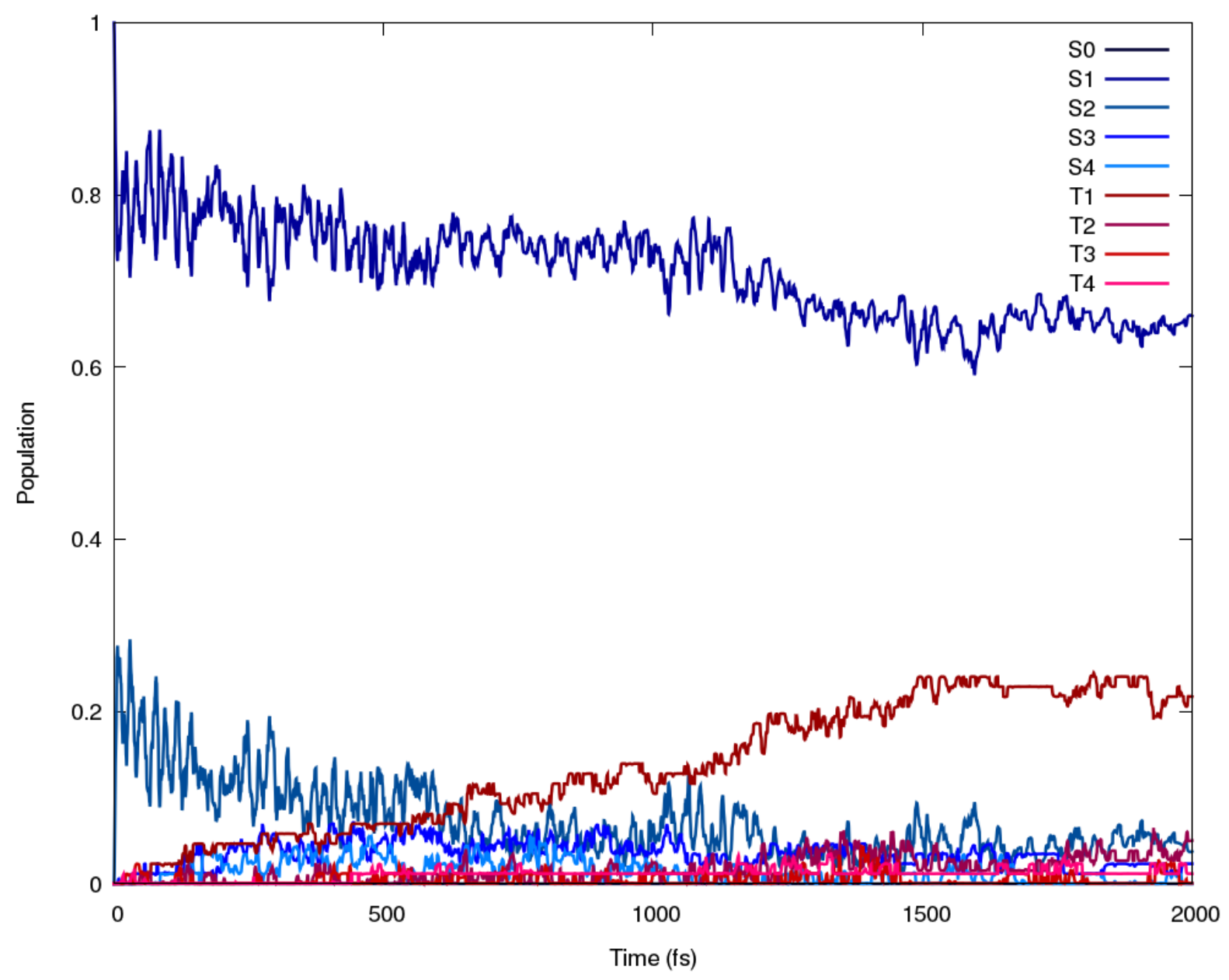

Figure S4. Evolution of the population of the states during the SH dynamic obtained from the multiconfiguration quantum amplitudes, triplet states are represented in red tones, convoluted for the ensemble of the trajectories, and for all the involved states. 


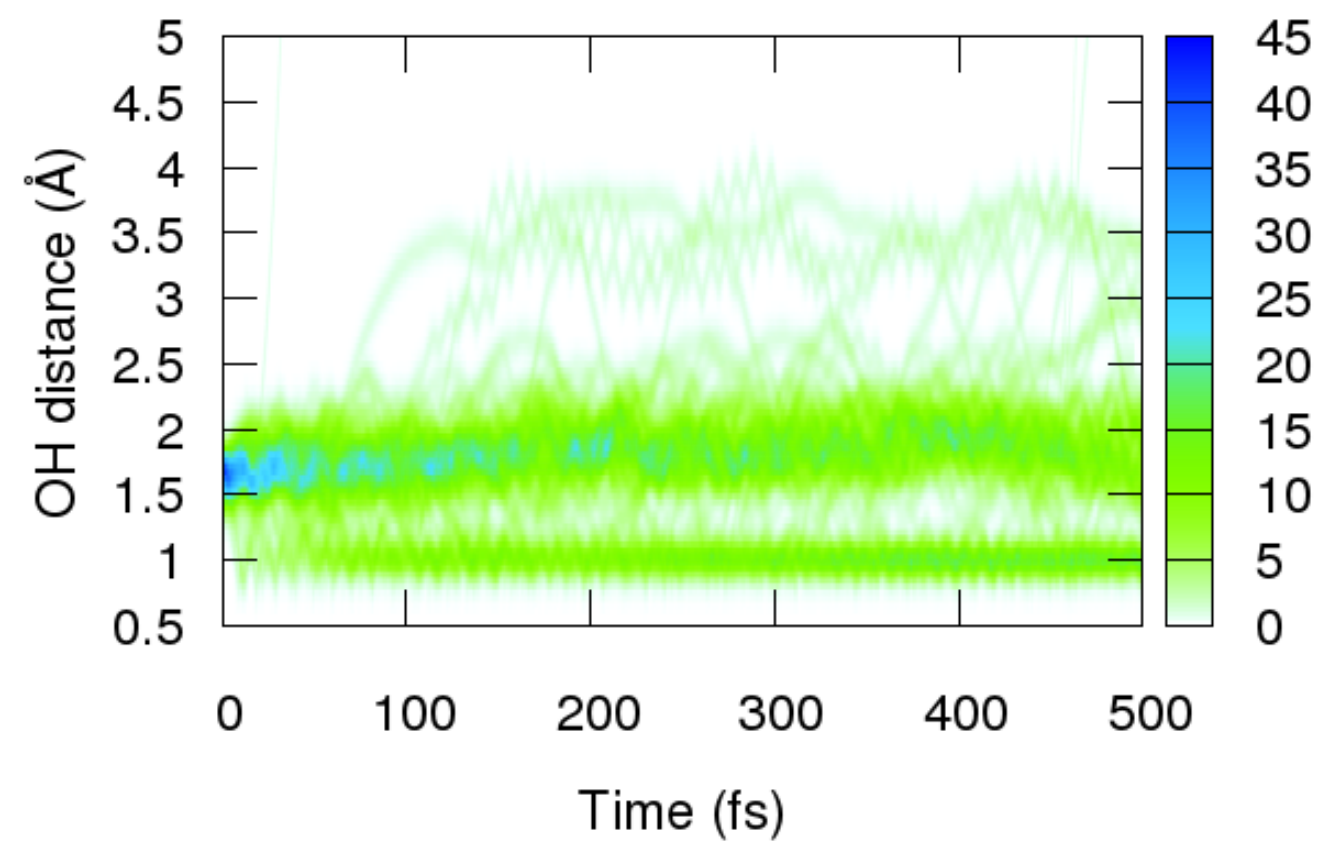

Figure S5. Evolution of the $\mathrm{OH}$ distance $\mathrm{d}$ for the ensemble of the trajectories during the first 500 fs of the SH dynamics.

\begin{tabular}{c|ccccccccc} 
& $\mathbf{S}_{\mathbf{0}}$ & $\mathbf{S}_{\mathbf{1}}$ & $\mathbf{S}_{\mathbf{2}}$ & $\mathbf{S}_{\mathbf{3}}$ & $\mathbf{S}_{\mathbf{4}}$ & $\mathbf{T}_{\mathbf{1}}$ & $\mathbf{T}_{\mathbf{2}}$ & $\mathbf{T}_{\mathbf{3}}$ & $\mathbf{T}_{\mathbf{4}}$ \\
\hline $\mathbf{S}_{\mathbf{0}}$ & 0 & 0 & 0 & 0 & 0 & 0 & 0 & 0 & 0 \\
$\mathbf{S}_{\mathbf{1}}$ & 0 & 0 & 6 & 1 & -9 & 0 & -1 & -8 & -18 \\
$\mathbf{S}_{\mathbf{2}}$ & 0 & -6 & 0 & 7 & -1 & 0 & 0 & 1 & 2 \\
$\mathbf{S}_{\mathbf{3}}$ & 0 & -1 & -7 & 0 & -10 & 0 & 0 & 0 & 0 \\
$\mathbf{S}_{\mathbf{4}}$ & 0 & 9 & 1 & 10 & 0 & 0 & 0 & 0 & 0 \\
$\mathbf{T}_{\mathbf{1}}$ & 0 & 0 & 0 & 0 & 0 & 0 & 23 & -6 & 0 \\
$\mathbf{T}_{\mathbf{2}}$ & 0 & 1 & 0 & 0 & 0 & -23 & 0 & 26 & 1 \\
$\mathbf{T}_{\mathbf{3}}$ & 0 & 8 & -1 & 0 & 0 & 6 & -26 & 0 & 14 \\
$\mathbf{T}_{\mathbf{4}}$ & 0 & 18 & -2 & 0 & 0 & 0 & -1 & -14 & 0 \\
\hline
\end{tabular}

Table S1. Matrix summing the differences of the hops between the states obtained on the ensemble of the SH trajectories. 